\title{
SOME EQUIVALENCE CONDITIONS FOR THE UNIFORM CONVERGENCE IN DISTRIBUTION OF SEQUENCES OF STOCHASTIC PROCESSES
}

\author{
BY \\ ERNEST G. KIMME
}

0 . Introduction. Let $\left(\Omega_{\omega}, ß, p\right)$ be a probability space and let $\left\{x_{n}(t, \omega), t \in T\right\}$ be a sequence, $n=1,2, \cdots$, of real stochastic processes defined thereon; $T$ is a real parameter set, and will usually here be taken to be the interval $[0,1]$. In [3], the convergence, as $n \rightarrow \infty$, of such a sequence was investigated for the case wherein the processes have independent increments, and a convergence criterion was given that insured the convergence in distribution of the sequence of random vectors

$$
\begin{aligned}
& \left\{\sup \left\{x_{n}(t, \omega), t_{j-1}<t \leqq t_{j}\right\}, 1 \leqq j \leqq N,\right. \\
& \left.\quad \inf \left\{x_{n}(t, \omega), t_{j-1}<t \leqq t_{j}\right\}, 1 \leqq j \leqq N\right\}_{n \geqq 1}
\end{aligned}
$$

for the case $t_{j}=j / N, 1 \leqq j \leqq N, T=[0,1]$, and $t_{0}=0$. The question of the convergence of these vectors is of the greatest importance in the consideration of the general problem of the convergence of a sequence $\left\{F\left[x_{n}\right], n \geqq 1\right\}$ of functionals defined on the processes $\left\{x_{n}(t, \omega), t \in T\right\}$. It is, accordingly, a matter of some interest to determine minimal conditions under which the sequence (1) will converge. It is the object of this paper to show some equivalences of such convergence conditions.

The convergence in distribution of the finite-dimensional distribution or characteristic functions of the process sequence $\left\{x_{n}(t, \omega), t \in T\right\}$ determines, uniquely in distribution only, a "limiting" process $\{x(t, \omega), t \in T\}$ whose finite-dimensional distribution or characteristic functions are the limits of the corresponding finite-dimensional distribution or characteristic functions of the sequence $\left\{x_{n}(t, \omega), t \in T\right\}$. This limiting process may without loss of generality be assumed to be separable. The criterion of separability is discussed fully in [1], and the remarks above are dealt with in [3]. Of interest here is the obvious implication that any stronger convergence condition on the finite-dimensional analytics of the process sequence $\left\{x_{n}(t, \omega), t \in T\right\}$ also determines, uniquely in distribution at least, a (separable) limiting process. In particular, a limiting process is so determined when the process sequence converges "uniformly in distribution." This form of convergence is defined in [3] (definition 2 of that reference) and is stated as follows: The sequence

Presented to the Society, August 29, 1958 under the title Note on the convergence of sequences of stochastic processes; received by the editors June 22, 1959. 
$\left\{x_{n}(t, \omega), t \in T\right\}$ of stochastic processes converges UNIFORMLY IN DISTRIBUTION if and only if for every integer $m \geqq 1$ and every positive $M$

$$
\lim _{n \rightarrow \infty} E\left\{\exp i \sum_{k=1}^{m} \mu_{k} x_{n}\left(t_{k}, \omega\right)\right\}
$$

uniformly for $\left(t_{1}, \cdots, t_{m} ; \mu_{1}, \cdots, \mu_{m}\right) \in T^{m} \otimes[-M, M]^{m}$.

In the sequel, the first result exhibits the manner in which this uniformity affects the finite-dimensional distributions of the process sequence. A more satisfying "continuity" theorem for uniform convergence in distribution is also established. It is then shown that the Theorem 7 of [3] dealing with the convergence of the sequence (1) can be improved to render the convergence uniform with respect of the partition $\left(t_{1}, \cdots, t_{N}\right)$ of $[0,1]$; this in turn is easily seen to imply the original hypothesis of uniform convergence in distribution. Finally, for processes constructed from "Poisson" arrays of rowindependent asymptotically infinitesimal random variables, these various convergence phenomena are shown to be equivalent to the uniform convergence with respect to $t \in T$ of the Lévy-Khintchine representations of certain "accompanying distributions." This last supplies the necessity of the conditions of Theorem 5 of [3], wherein only the sufficiency was shown. From this series of theorems it therefore appears that the hypothesis of uniform convergence in distribution is of much greater significance to the general problem of the convergence of functionals on process sequences than is indicated in [3]. It is one purpose of this paper to exhibit this point.

The notation of this paper will be that of [3]:

$$
\begin{gathered}
p\left\{x_{n}\left(t_{j}, \omega\right) \leqq \lambda_{j}, 1 \leqq j \leqq m\right\}=F_{n}\left(t_{1}, \cdots, t_{m} ; \lambda_{1}, \cdots, \lambda_{m}\right), \\
E\left\{\exp i \sum_{k=1}^{m} \mu_{k} x_{n}\left(t_{k}, \omega\right)\right\}=\phi_{n}\left(t_{1}, \cdots, t_{m} ; \mu_{1}, \cdots, \mu_{m}\right)
\end{gathered}
$$

and similarly for the "limiting process" $\{x(t, \omega), t \in T\}$, dropping the index $n$ throughout. The $n$-fold integral mean of an $n$-dimensional distribution $F\left(x_{1}, \cdots, x_{n}\right)$ over the interval $\otimes_{k-1}^{n}\left[x_{k}-h, x_{k}+h\right]$ will be denoted by $F^{(h)}\left(x_{1}, \cdots, x_{n}\right)=\int_{-h}^{h} \cdots(n) \cdots \int_{-h}^{h} \frac{F\left(x_{1}+u_{1}, \cdots, x_{n}+u_{n}\right) d u_{1} \cdots d u_{n}}{(2 h)^{n}}$ and the corresponding characteristic functions by

$$
\phi^{(h)}\left(\mu_{1}, \cdots, \mu_{n}\right)=\left(\prod_{k=1}^{n} \frac{\sin \mu_{k} h}{\mu_{k} h}\right) \phi\left(\mu_{1}, \cdots, \mu_{n}\right),
$$

where $\phi$ is the characteristic function of $F$.

The proofs of some of our results are at times quite tedious, and an effort has been made to avoid details wherever the analysis was felt to be straight- 
forward enough to warrant it. A great many elementary details remain where it seems needful to exercise some caution in the order in which things are done.

1. "Continuity" theorems. In this section some equivalences are established between convergence of sequences of finite-dimensional distributions and uniform convergence in distribution of the related stochastic processes. The first result is obtained in the absence of any condition other than that of uniform convergence in distribution.

TheOREM 1. Let $\left\{x_{n}(t, \omega), t \in T\right\}$ be a sequence of stochastic processes converging uniformly in distribution. Then for every positive integer $m$ and every $\delta>0$ and $h>0$,

$$
\exists \lim _{n \rightarrow \infty} \int_{\left|\lambda_{j}-\lambda_{0 j}\right|<\delta_{j} ; 1 \leq j \leq m} d F_{n}^{(h)}\left(t_{1}, \cdots, t_{m} ; \lambda_{1}, \cdots, \lambda_{m}\right)
$$

uniformly in $\left\{t_{1}, \cdots, t_{m}\right\} \in T^{n}$, in $\left\{\delta_{1}, \cdots, \delta_{m}\right\} \in(0, \delta)^{m}$, and in $\left\{\lambda_{01}, \cdots, \lambda_{0 m}\right\} \in(-\infty, \infty)^{m}$. Moreover, the limit is given by

$$
\int_{\left|\lambda_{j}-\lambda_{0 j}\right|<\delta_{j} ; 1 \leqq j \leqq m} d F^{(h)}\left(t_{1}, \cdots, t_{m} ; \lambda_{1}, \cdots, \lambda_{m}\right)
$$

where $F^{(h)}(t ; \lambda)$ is the integral mean over $[-h, h]^{m}$ of $F(t ; \lambda)$, this last being a finite-dimensional distribution of the limiting process.

Proof. The Lévy inversion formula for $F_{n}^{(h)}$ reads

$$
\begin{aligned}
A_{n}= & \int_{\left|\lambda_{j}-\lambda_{0 j}\right|<\delta_{j} ; 1 \leq j \leq m} d F_{n}^{(h)}\left(t_{1}, \cdots, t_{m} ; \lambda_{1}, \cdots, \lambda_{m}\right) \\
= & \frac{1}{\pi^{m}} \int_{-\infty}^{\infty} \cdots(m) \cdots \int \exp \left(-i \sum_{k=1}^{m} \mu_{k} \lambda_{0 k}\right) \prod_{k=1}^{m}\left(\frac{\sin \mu_{k} \delta_{k}}{\mu_{k}} \cdot \frac{\sin \mu_{k} h}{\mu_{k} h}\right) \\
& \cdot \phi_{n}\left(t_{1}, \cdots, t_{m} ; \mu_{1}, \cdots, \mu_{m}\right) d \mu_{1} \cdots d \mu_{m} .
\end{aligned}
$$

Applying the estimate

$$
\frac{\left|\sin \mu_{k} \delta_{k} \cdot \sin \mu_{k} h\right|}{\mu_{k}^{2} h} \leqq \frac{1+h \delta_{k}}{h} \cdot \frac{1}{1+\mu_{k}^{2}}
$$

it is easily seen that

$$
\left|A_{n_{1}}-A_{n_{2}}\right| \leqq \frac{B_{n_{1}, n_{2}}}{\pi^{m}}\left(\prod_{k=1}^{m} \frac{1+h \delta_{k}}{h}\right)\left(\int_{-\infty}^{\infty} \frac{(1+|\mu|)^{1 / 2}}{1+\mu^{2}} d \mu\right)^{m}
$$

where

$$
B_{n_{1, \eta_{2}}}=\sup \left\{\frac{\left|\phi_{n_{1}}(t ; \mathfrak{u})-\phi_{n_{2}}(t ; \mathfrak{u})\right|}{\prod_{k=1}^{m}\left(1+\left|\mu_{k}\right|\right)^{1 / 2}}: t \in T^{m}, \mathfrak{u} \in(-\infty, \infty)^{m}\right\} .
$$


The hypothesis of uniform convergence in distribution of the process sequence $\left\{x_{n}(t, \omega), t \in T\right\}$ and the presence of the factors $\left(1+\left|\mu_{k}\right|\right)^{1 / 2}$ in the denominator of the supremands of $B_{n_{1}, n_{2}}$ insure that $B_{n_{1}, n_{2}} \rightarrow 0$ as $n_{1}, n_{2} \rightarrow \infty$ independently. The second factor in the estimate above of $\left|A_{n_{1}}-A_{n_{2}}\right|$ is less than or equal to

$$
\left(\frac{1+\delta h}{h}\right)^{m}
$$

and the third factor in the same estimate is independent of $t$ and $\lambda_{0}$. The first conclusion of Theorem 1 is then established. The second conclusion follows immediately from the observations made in the introduction concerning the relations between $F, F^{(h)}, \phi$, and $\phi^{(h)}$ and the remark that uniform convergence in distribution implies ordinary convergence in distribution of stochastic processes.

In order to augment the hypothesis of Theorem 1 to the point where an equivalence theorem can be established, it is necessary to state a theorem of the "Helly convergence" type dealing with convergence uniformly with respect to parameters not involved in the Lebesgue-Stieltjes integration. We will state without proof the theorem (which for lack of anything better we have called the "Uniform Helly Convergence Theorem") in the multidimensional form:

Uniform Helly Convergence Theorem. Let $T$ and $M$ be any two ( $a b$ stract) sets. Denote $(-\infty, \infty)^{m}$ (for $m$ a positive integer) by $R$. Let $\left\{f_{n}(t ; x) ; n \geqq 0\right\}$ be a sequence of real functions defined on $T \otimes R$, satisfying the following conditions:

(a) $\exists V_{0}>0$ such that for all $n \geqq 0$ and all $t \in T$,

$$
\operatorname{Var}\left\{f_{n}(t ; \mathbf{x}) ; \mathbf{x} \in R\right\} \leqq V_{0} .
$$

(b) For any $\epsilon>0$ there exists a positive number $\Lambda_{\epsilon}>0$, depending only on $\epsilon$, so large that

$$
\operatorname{Var}\left\{f_{n}(t ; \mathrm{x}) ; \mathrm{x} \in R-\left[-\Lambda_{\epsilon}, \Lambda_{\epsilon}\right]\right\} \leqq \epsilon
$$

for all $n \geqq 0$ and all $t \in T$.

(c) For every $h>0$ and each $\mathrm{x} \in R$,

$$
\lim _{n \rightarrow \infty} f_{n}^{(h)}(t ; \mathbf{x})=f_{0}^{(h)}(t ; \mathbf{x})
$$

uniformly for $t \in T$; the superscript denotes the integral mean of $f_{n}$ on $[-h, h]^{m}$ with respect to the second (vector) variable $\mathrm{x}$, as defined in the introduction. Then for any real or complex function $g(\mu ; \mathrm{x})$ defined and bounded on $M \otimes R$, and such that for each $\mathrm{x} \in R$,

$$
\lim _{\mathbf{x}^{\prime} \rightarrow \mathbf{x}} g\left(\mu ; \mathbf{x}^{\prime}\right)=g(\mu ; \mathbf{x})
$$


uniformly in $\mu \in M$, the relation

$$
\lim _{n \rightarrow \infty} \int_{\{\mathbf{x} \in R\}} g(\mu ; \mathbf{x}) d f_{n}(t ; \mathbf{x})=\int_{\{\mathbf{x} \in R\}} g(\mu ; \mathbf{x}) d f_{0}(t ; \mathbf{x})
$$

holds uniformly in $(\mu, t) \in M \otimes T$.

Using this rather formidable result we can establish a direct generalization of the "continuity" theorem for characteristic functions. The convergence we are concerned with is, of course, uniform convergence in distribution of sequences of stochastic processes, and the continuity condition of the limiting characteristic function in the one-dimensional case carries over in an obvious way; however, this continuity condition now imposes some uniformity conditions on the finite-dimensional distributions of the limiting process.

TheOREM 2. Let $\left\{x_{n}(t, \omega), t \in T\right\}$ be a sequence of stochastic processes. Then a necessary and sufficient condition that for each positive $h$

(i) $\lim _{n \rightarrow \infty} F_{n}^{(h)}\left(t_{1}, \cdots, t_{m} ; \lambda_{1}, \cdots, \lambda_{m}\right)$ exists for each integer $m \geqq 1$ uniformly for $\left(t_{1}, \cdots, t_{m} ; \lambda_{1}, \cdots, \lambda_{m}\right) \in T^{m} \otimes[-\infty, \infty]^{m}$, and

(ii) for each $\epsilon>0$ there exist $\Lambda_{\epsilon}>0$ independent of $t \in T$ such that

$$
\int_{|\lambda|>\Lambda_{\epsilon}} d F^{(h)}(t ; \lambda)<\epsilon
$$

where

$$
F^{(h)}(t ; \lambda)=\lim _{n} F_{n}^{(h)}(t ; \lambda)
$$

is that

$\left(\mathrm{i}^{\prime}\right)$ the processes $\left\{x_{n}(t, \omega), t \in T\right\}$ converge uniformly in distribution, and

(ii') $\lim _{|\mu| \rightarrow 0} \phi(t ; \mu)=1$ uniformly in $t \in T$, where $\phi(t ; \mu)=\lim _{n} \phi_{n}(t ; \mu)$.

Proof. (ii) $\Rightarrow$ (ii'). If $h / \pi$ is not an odd multiple of $\mu$, then the definition of $\phi^{(h)}(t ; \mu)$ implies

$$
|1-\phi(t ; \mu)| \leqq\left|1-\frac{\mu h}{\sin \mu h}\right|+\left|\frac{\sin \mu h}{\mu h}\right| \cdot\left|1-\phi^{(h)}(t ; \mu)\right| .
$$

The second factor in the second term can be estimated for any $\Lambda>0$ by

$$
\begin{aligned}
\left|1-\phi^{(h)}(t ; \mu)\right| & =\left|\int_{-\infty}^{\infty}\left(1-e^{i \mu x}\right) d F^{(h)}(t, x)\right| \\
& \leqq 2 \int_{|x|>\Lambda} d F^{(h)}(t ; x)+|\mu| \Lambda .
\end{aligned}
$$

If then $\mu$ is chosen so small that 


$$
|\mu| \Lambda<\int_{|x|>\Lambda} d F^{(h)}(t ; x) \leqq 1
$$

we find

$$
\left|1-\phi^{(h)}(t ; \mu)\right|<3 \int_{|x|>\Lambda} d F^{(h)}(t ; \mu) .
$$

Since (ii) implies that the integral on the right in the last inequality can be made small uniformly in $t$ for sufficiently large $\Lambda$, and since

$$
\lim _{|\mu| \rightarrow 0} \frac{\mu h}{\sin \mu h}=1
$$

obviously independently of $t$, we conclude that (ii') holds whenever (ii) holds.

$\left(\mathrm{ii}^{\prime}\right) \Rightarrow(\mathrm{ii})$. From I: (11.8) of [1] it follows that

$$
\int_{|x|>\Lambda} d F^{(h)}(t ; x) \leqq(1+2 \pi)^{2} \Lambda \int_{0}^{1 / \Lambda}\left|1-\phi^{(h)}(t ; \mu)\right| d \mu
$$

which indicates that $\left(\mathrm{ii}^{\prime}\right) \Rightarrow(\mathrm{ii})$, and hence (ii) and (ii') are equivalent independently of the rest of the theorem.

The necessity of Theorem 2 follows now from the Uniform Helly Convergence Theorem, while the sufficiency follows from Theorem 1.

The conditions (ii) and (ii') of Theorem 2 suggest that if the parameter set $T$ were compact, some form of stochastic continuity on the limiting process might replace these uniformity requirements. Such a result is Theorem 3.

TheOREM 3. Let $\left\{x_{n}(t, \omega), t \in T\right\}$ be a sequence of stochastic processes with compact parameter set $T$. Let $\{x(t, \omega), t \in T\}$ be a real stochastic process which is continuous in distribution, i.e., such that

$$
\lim _{t^{\prime} \rightarrow t ; t^{\prime} \in \boldsymbol{T}} F\left(t^{\prime} ; \lambda\right)=F(t ; \lambda)
$$

at continuity points $\lambda$ of the limit, for each $t \in T$. Then a necessary and sufficient condition that $\left\{x_{n}(t, \omega), t \in T\right\}$ converge uniformly in distribution to $\{x(t, \omega), t \in T\}$ is that for each integer $m \geqq 1$ and each $h>0$

$$
\lim _{n \rightarrow \infty} F_{n}^{(h)}\left(t_{1}, \cdots, t_{m} ; \lambda_{1}, \cdots, \lambda_{m}\right)=F^{(h)}\left(t_{1}, \cdots, t_{m} ; \lambda_{1}, \cdots, \lambda_{m}\right)
$$

uniformly in $\left(t_{1}, \cdots, t_{m} ; \lambda_{1}, \cdots, \lambda_{m}\right) \in T \otimes(-\infty, \infty)^{m}$.

Proof. The proof is immediate from Theorem 2 and the hypothesis of compactness (sequential) of $T$. We omit the details.

The addition of an independence condition (in the form of the assumption that the processes $\left\{x_{n}(t, \omega), t \in[0,1]\right\}$ have independent increments) reduces the multidimensional convergence conditions of the preceding theorems to 
one-dimensional form. The reasons are obvious; by such an hypothesis we impose an explicit analytic relation between joint and "marginal" distributions of the random variables of the relevant processes. It is an interesting, and as yet open, question whether less stringent dependence relations will still permit results like Theorem 4 following.

THEOREM 4. Let $\left\{x_{n}(t, \omega), t \in T\right\}$ be a sequence of stochastic processes with independent increments and let $T$ be a compact set. Let $\{x(t, \omega), t \in T\}$ be a stochastic process with independent increments which is stochastically continuous in the sense of Theorem 3 above. Then a necessary and sufficient condition that $\left\{x_{n}(t, \omega), t \in T\right\}$ converge uniformly in distribution to $\left\{x_{n}(t, \omega), t \in T\right\}$ is that for each $h>0$

$$
\lim _{n \rightarrow \infty} F_{n}^{(h)}(t ; \lambda)=F^{(h)}(t ; \lambda)
$$

uniformly for $(t, \lambda) \in T \otimes(-\infty, \infty)$.

This last result is in essence Theorem 3 of [3], and for proof we refer thereto. The present Theorem 4 is included primarily for continuity of exposition.

Before concluding $\S 1$, it will be convenient to establish two further results dealing with uniform convergence in distribution. The first of these, Theorem 5 following, exhibits an alternative formulation of the uniform convergence of smoothed distributions and holds quite generally. It is, moreover, clear from Theorem 5 that the "Lévy distance" between distributions could be extended and used to obtain yet another formulation of this same property.

THEOREM 5.

$$
\lim _{n \rightarrow \infty} F_{n}^{(h)}\left(t_{1}, \cdots, t_{m} ; x_{1}, \cdots, x_{m}\right)=F^{(h)}\left(t_{1}, \cdots, t_{m} ; x_{1}, \cdots, x_{m}\right)
$$

for every $h>0$ uniformly for $(t ; x) \in T^{m} \otimes(-\infty, \infty)^{m}$ if and only if for every $\epsilon>0$ there exists a positive integer $N$, depending only on $m$ and $\epsilon$, so large that $n \geqq N \Longrightarrow$

$$
\begin{aligned}
F\left(t_{1}, \cdots, t_{m} ; x_{1}-\epsilon, \cdots, x_{m}-\epsilon\right) & -\epsilon \leqq F_{n}\left(t_{1}, \cdots, t_{m} ; x_{1}, \cdots, x_{m}\right) \\
& \leqq F\left(t_{1}, \cdots, t_{m} ; x_{1}+\epsilon, \cdots, x_{m}+\epsilon\right)+\epsilon
\end{aligned}
$$

for all $(t, x) \in T^{m} \otimes(-\infty, \infty)^{m}$.

Proof. The smoothed distributions $F^{(h)}$ satisfy the inequalities

$$
\begin{aligned}
F^{(h)}\left(t_{1}, \cdots, t_{m} ; x_{1}-h, \cdots, x_{m}-h\right) & \geqq F\left(t_{1}, \cdots, t_{m} ; x_{1}, \cdots, x_{m}\right) \\
& \leqq F^{(h)}\left(t_{1}, \cdots, t_{m} ; x_{1}+h, \cdots, x_{m}+h\right)
\end{aligned}
$$

identically in $h, t$, and $x$, and similarly for $F_{n}$. The required implications of the theorem follow in the obvious way. 
The "stochastic continuity" needed in Theorems 3 and 4 is equivalent, when the limiting process has independent increments, to the much stronger condition of absence of fixed points of discontinuity. We refer to [1, III, Theorem 2.8]. In the present circumstances it would be very convenient to be able to replace this continuity condition on the limiting process by a condition on the process sequence itself. As might be expected, the condition that does this for us looks like an equicontinuity condition on the process sequence $\left\{x_{n}(t, \omega), t \in[0,1]\right\}, n \geqq 1$. Theorem 6 following makes this precise; the corollary weakens the equicontinuity requirement in a very pleasant way using, again, the hypothesis of independent increments in an essential way.

Theorem 6. Let $\left\{x_{n}(t, \omega), t \in[0,1]\right\}$ be a sequence of stochastic processes with independent increments converging uniformly in distribution. Then (any separable version of) the limiting process has independent increments. Moreover, (any separable version of) the limiting process has no fixed points of discontinuity if and only if the following additional condition on the convergence of $\left\{x_{n}(t, \omega), t \in[0,1]\right\}$ is satisfied: for each $\epsilon>0$ there exist a positive integer $n_{\epsilon}$ and a positive number $\delta_{\epsilon}$ such that if $n \geqq n_{\epsilon}$ and $0=\tau_{0}<\tau_{1}<\cdots<\tau_{N}=1$ is any partition of $[0,1]$ such that

then

$$
\max \left\{\tau_{k}-\tau_{k-1}: 1 \leqq k \leqq N\right\}<\delta_{\epsilon}
$$

$$
\sup \left\{p\left\{\left|x_{n}(t, \omega)-x_{n}\left(\tau_{k}, \omega\right)\right| \geqq \epsilon\right\}: \tau_{k-1} \leqq t \leqq \tau_{k}, 1 \leqq k \leqq N\right\}<\epsilon .
$$

Proof. The first part of Theorem 6 is established (under the weaker hypothesis of ordinary convergence in distribution) as the corollary of Theorem 2 of [3]. From remarks made earlier it also follows that we need to show that $(6.1)$ is equivalent to the continuity of $\phi(t ; \mu)=\lim _{n} \phi_{n}(t ; \mu)$ for $(t ; \mu)$ $\in[0,1] \otimes[-M, M]$ for any $M>0$. The proof proceeds, therefore, using the characteristic functions of the various processes. The condition (6.1) has the following analogue in terms of characteristic functions: for any $\epsilon>0$ and $M>0$ there exist a positive integer $n_{\epsilon}^{\prime}$ and a positive number $\delta_{\epsilon}^{\prime}$ (depending in general on $M$ ) for which, for any ordered partition $\left\{\tau_{k}\right\}$ of $[0,1]$ such that

$$
\max \left\{\tau_{k}-\tau_{k-1}: 1 \leqq k \leqq N\right\}<\delta_{\epsilon}^{\prime},
$$

we have, for $n>n_{\epsilon}$,

$$
\sup \left\{\left|\phi_{n}(t ; \mu)-\phi_{n}\left(\tau_{k} ; \mu\right)\right|: \tau_{k-1} \leqq t \leqq \tau_{k}, 1 \leqq k \leqq N,|\mu| \leqq M\right\}<\epsilon .
$$

We can show that $(6.1) \Leftrightarrow(6.2) \Leftrightarrow \lim _{n} \phi_{n}(t ; \mu)$ is continuous.

In outline, we show that $(6.1) \Rightarrow(6.2) \Rightarrow \lim _{n} \phi_{n}(t ; \mu)$ is continuous $\Rightarrow(6.2)$ $\Rightarrow(6.1)$ in that order. Since for $\left\{\tau_{k}\right\}$ as defined above, we have

$$
\left|\phi_{n}(t ; \mu)-\phi_{n}\left(\tau_{k} ; \mu\right)\right|<2 p\left\{\left|x_{n}(t ; \omega)-x_{n}\left(\tau_{k}, \omega\right)\right|>\Lambda\right\}+|\mu| \Lambda,
$$


for any $k, t, \mu, n, \Lambda>0$, the first implication, $(6.1) \Rightarrow(6.2)$ follows by suitable choice of $\Lambda$ small and $n$ large. If $(6.2)$ holds, $\lim _{n} \phi_{n}(t ; \mu)$ is continuous, since $\lim _{n} \phi_{n}(t ; \mu)=\phi(t ; \mu)$ uniformly for $(t, \mu) \in[0,1] \otimes[-M, M]$ by the hypothesis of uniform convergence in distribution, (6.2) implies asymptotic equicontinuity of $\phi_{n}(t ; \mu)$, and $\phi(t ; \mu)$ is a characteristic function. If, conversely, $\lim _{n} \phi_{n}(t ; \mu)$ is continuous, the uniform convergence of $\phi_{n}$ to $\phi$ implies (6.2) immediately. Finally, $(6.2) \Rightarrow(6.1)$ since it is easily shown that if

$$
0<C \leqq \inf \{|\phi(t ; \mu)|:(t ; \mu) \in[0,1] \otimes[-M, M]\}
$$

then

$$
\begin{aligned}
& p\left\{\left|x_{n}(t, \omega)-x_{n}\left(\tau_{k}, \omega\right)\right| \geqq \epsilon\right\} \\
& \leqq \frac{(M+2 \pi / \epsilon)^{2}}{M^{2}} \cdot C \cdot \sup \left\{\left|\phi_{n}(t ; \mu)-\phi_{n}\left(\tau_{k} ; \mu\right)\right|: \tau_{k-1} \leqq t \leqq \tau_{k}, 1 \leqq k \leqq n,\right. \\
& |\mu| \leqq M\} .
\end{aligned}
$$

That such a number $C$ exists follows from (6.2), since (6.2) insures the continuity of $\phi$; this, together with the conclusion that $\{x(t ; \omega), t \in[0,1]\}$ has independent increments implies that $\phi$ is not only continuous but is an infinitely divisible characteristic function, and is therefore bounded away from zero on sets $[0,1] \otimes[-M, M]$. Theorem 6 is established.

TheORem 6, Corollary. Theorem 6 holds with condition (6.1) replaced by the following: there exists a sequence of numbers $\left\{\hat{\delta}_{n}, n \geqq 1\right\}$ such that

$$
\lim _{n \rightarrow \infty} \hat{\delta}_{n}=0
$$

and for every $\epsilon>0$

$(6.1)^{\prime \prime} \lim _{n \rightarrow \infty}$

$$
\left.|t-s|<\hat{\delta}_{n}, 0 \leqq t, s \leqq 1\right\}=0 .
$$

Proof. Since $(6.1) \Rightarrow(6.1)^{\prime \prime}$ trivially, it is necessary to show only that under the hypothesis of Theorem $6,(6.1)^{\prime \prime} \Rightarrow(6.1)$. To see this we observe that since the processes $\left\{x_{n}(t, \omega), t \in[0,1]\right\}$ have independent increments

$$
p\left\{\left|x_{n}(t, \omega)-x_{n}(s, \omega)\right| \geqq \epsilon\right\}
$$

is a nondecreasing function of $|t-s|$, and

$$
\exists \lim _{n \rightarrow \infty} p\left\{\left|x_{n}(t, \omega)-x_{n}(s, \omega)\right| \geqq \epsilon\right\}
$$

at continuity points of the limit. In fact, the hypothesis of uniformity on the convergence of $\left\{x_{n}(t, \omega), t \in[0,1]\right\}$ permits the following assertion: for any $\eta>0$ there exists an integer $N_{\eta}$ such that for all $(t, s) \in[0,1]^{2}$, any $\epsilon>0$. and all $m, n \geqq N_{\eta}$ 


$$
\begin{aligned}
p\left\{\left|x_{m}(t, \omega)-x_{m}(s, \omega)\right| \geqq \epsilon\right. & +\eta\}-\eta \\
& \leqq p\left\{\left|x_{n}(t, \omega)-x_{n}(s, \omega)\right| \geqq \epsilon\right\} \\
& \leqq p\left\{\left|x_{m}(t, \omega)-x_{m}(s, \omega)\right| \geqq \epsilon-\eta\right\}+\eta ;
\end{aligned}
$$

this follows in the manner of Theorem 5. Let $\left\{\tau_{k}, 1 \leqq k \leqq N\right\}$ be any ordered partition of $[0,1]$. Let $\epsilon>0$ be fixed and choose $n_{1}$ so large that $n \geqq n_{1} \Rightarrow$

$$
\sup \left\{p\left\{\left|x_{n}(t, \omega)-x_{n}(s, \omega)\right| \geqq \epsilon / 2\right\} ;|t-s|<\hat{\delta}_{n}\right\}<\epsilon / 2 .
$$

Let $n_{2}=\max \left\{n_{1}, N_{\epsilon / 2}\right\}$; then (6.3) holds for $\eta=\epsilon / 2$ and $m, n \geqq n_{2}$. We may now write for $n \geqq n_{2}$ and $\tau_{k-1} \leqq t \leqq \tau_{k}$

$$
\begin{aligned}
p\left\{\left|x_{n}(t, \omega)-x_{n}\left(\tau_{k}, \omega\right)\right| \geqq \epsilon\right\} & \leqq p\left\{\left|x_{n}\left(\tau_{k}, \omega\right)-x_{n}\left(\tau_{k-1}, \omega\right)\right| \geqq \epsilon\right\} \\
& \leqq p\left\{\left|x_{n_{2}}\left(\tau_{k}, \omega\right)-x_{n_{2}}\left(\tau_{k-1}, \omega\right)\right| \geqq \epsilon / 2\right\}+\epsilon / 2 .
\end{aligned}
$$

Upon setting $\delta_{\epsilon}$ of Theorem 6 equal to $\hat{\delta}_{n_{2}}$, we find that if

$$
\max \left\{\tau_{k}-\tau_{k-1} ; 1 \leqq k \leqq N\right\}<\hat{\delta}_{n_{2}}=\delta_{\epsilon}
$$

we have for $n \geqq n_{2}$ that

$$
\begin{aligned}
& \sup \left\{p\left\{\left|x_{n}(t, \omega)-x_{n}\left(\tau_{k}, \omega\right)\right| \geqq \epsilon\right\} ; \tau_{k-1} \leqq t \leqq \tau_{k}, 1 \leqq k \leqq N\right\} \\
& \leqq \max _{1 \leqq k \leqq N} p\left\{\left|x_{n_{2}}\left(\tau_{k}, \omega\right)-x_{n_{2}}\left(\tau_{k-1}, \omega\right)\right| \geqq \epsilon / 2\right\}+\epsilon / 2 \\
& \leqq \sup \left\{p\left\{\left|x_{n_{2}}(t, \omega)-x_{n_{2}}(s, \omega)\right| \geqq \epsilon / 2\right\} ;|t-s|<\hat{\delta}_{n_{2}}\right\}+\epsilon / 2 \\
&<\epsilon .
\end{aligned}
$$

This is (6.1) of Theorem 6, and the corollary follows.

2. Convergence properties of suprema and infima. As remarked in the introduction, the notion of uniform convergence in distribution of sequences of stochastic processes was formulated to obtain an extension of Donsker's result for functionals defined on the Wiener process. The crux of such an extension [3] was shown by Donsker (in the Gaussian case alluded to) to lie in the convergence in distribution of vectors of suprema and infima of the form

$$
\left\{\sup _{t_{j-1} \leqq t \leqq t_{j}} x_{n}(t, \omega), \inf _{t_{j-1} \leqq t \leqq t_{j}} x_{n}(t, \omega) ; 1 \leqq j \leqq N\right\}
$$

where $\left\{t_{j}\right\}_{N}$ is an ordered partition of the parameter set of the processes $\left\{x_{n}(t, \omega), t \in T\right\}$. The joint distributions of these vectors are probabilities of certain subsets of the function-space representation of these stochastic processes; these subsets are of the form

$$
\left\{\alpha_{j} \leqq x_{n}(t, \omega) \leqq \beta_{j}, t_{j-1} \leqq t \leqq t_{j}, 1 \leqq j \leqq N\right\} .
$$

It should be remarked that these subsets resemble the "compact-open" sets of the function-space. Theorem 7 following exhibits an equivalence between 
the convergence (with an accessory uniformity) of the probabilities of such sets and uniform convergence in distribution of the underlying processes. We quote first a lemma due in essence to Kolmogorov.

Lemma. If $\{y(t, \omega), t \in[0,1]\}$ is a separable stochastic process with independent increments and $0=t_{0}<t_{1}<\cdots<t_{N}=1$ and $0=\tau_{0}<\tau_{1}<\cdots<\tau_{M}$ are any two partitions of $[0,1]$ such that the second is a refinement of the first (i.e., for each $k$ there is a $p_{k}$ such that $t_{k}=\tau_{p_{k}}$ ), then for any $\epsilon>0$ and any real numbers $\left(\alpha_{1}, \cdots, \alpha_{N}, \beta_{1}, \cdots, \beta_{N}\right)$, we have

$$
\begin{array}{r}
p\left\{\alpha_{j}+\epsilon \leqq y\left(\tau_{k}, \omega\right) \leqq \beta_{j}-\epsilon ; k \ni t_{j-1}<\tau_{k} \leqq t_{j}, 1 \leqq j \leqq N\right\} \\
\leqq p\left\{\alpha_{j} \leqq y(t, \omega) \leqq \beta_{j} ; t_{j-1}<t \leqq t_{j}, 1 \leqq j \leqq N\right\} \\
\quad+\max _{1 \leqq k \leqq M} \sup _{\tau_{k-1} \leqq t \leqq \tau_{k}} p\left\{\left|y(t, \omega)-y\left(\tau_{k}, \omega\right)\right| \geqq \epsilon\right\} .
\end{array}
$$

We omit proof of this result here; it is proved (in a trivially different formulation) in Theorem 7 of [3].

THEOREM 7. Let $\left\{x_{n}(t, \omega), t \in[0,1]\right\}$ be a sequence of separable stochastic processes with independent increments. Let $\{x(t, \omega), t \in[0,1]\}$ be a separable stochastic process with independent increments and no fixed points of discontinuity. The sequence $\left\{x_{n}(t, \omega), t \in[0,1]\right\}$ converges uniformly in distribution to $\{x(t, \omega), t \in[0,1]\}$ if and only if for every positive integer $N$ and every $\epsilon>0$ there exists a positive integer $n(\epsilon, N)$ such that if $n \geqq n(\epsilon, N)$, then

$$
\begin{aligned}
p\left\{\alpha_{j}+\epsilon\right. & \left.\leqq x(t, \omega) \leqq \beta_{j}-\epsilon, t_{j-1}<t \leqq t_{j}, 1 \leqq j \leqq N\right\}-\epsilon \\
& \leqq p\left\{\alpha_{j} \leqq x_{n}(t, \omega) \leqq \beta_{j}, t_{j-1}<t \leqq t_{j}, 1 \leqq j \leqq N\right\} \\
& \leqq p\left\{\alpha_{j}-\epsilon \leqq x(t, \omega) \leqq \beta_{j}+\epsilon, t_{j-1}<t \leqq t_{j}, 1 \leqq j \leqq N\right\}+\epsilon
\end{aligned}
$$

for any partition $0=t_{0}<t_{1}<\cdots<t_{N}=1$ of $[0,1]$ and any $2 N$ numbers $\left(\alpha_{1}, \cdots, \alpha_{N}, \beta_{1}, \cdots, \beta_{N}\right)$.

Proof. We establish first that condition (7.1) is a necessary consequence of the convergence and continuity hypotheses. For notational convenience, let

$$
p\left(y,\left\{\tau_{k}\right\}, \epsilon\right)=\max _{1 \leqq k \leqq M} \sup _{\tau_{k-1}<t \leqq \tau_{k}} p\left\{\left|y(t, \omega)-y\left(\tau_{k}, \omega\right)\right| \geqq \epsilon\right\}
$$

for an stochastic process $\{y(t, \omega), t \in[0,1]\}$, any $\epsilon>0$ and any partition $0=\tau_{0}<\tau_{1}<\cdots<\tau_{M}=1$ for $[0,1]$. Let also

$$
\begin{aligned}
& A(T, \epsilon)=\left\{f \in(-\infty, \infty)^{[0,1]}: \alpha_{j}-\epsilon \leqq f(\tau) \leqq \beta_{j}+\epsilon\right. \\
& \text { for all } \left.\tau \in T \ni t_{j-1}<\tau \leqq \tau_{j} ; 1 \leqq j \leqq N\right\}
\end{aligned}
$$

for any real $\epsilon$ and any subset $T$ of $[0,1]$; for the purposes of this proof the dependence of the set $A$ on the numbers $\left\{t_{j}\right\},\left\{\alpha_{j}\right\},\left\{\beta_{j}\right\}$ need not be explicitly indicated. In this notation, (7.1) becomes 


$$
\begin{aligned}
p\{x \in A([0,1],-\epsilon)\}-\epsilon & \leqq p\left\{x_{n} \in A([0,1], 0)\right\} \\
& <p\{x \in A[(0,1], \epsilon)\}+\epsilon .
\end{aligned}
$$

The proof proceeds easily, depending on the preceding lemma and Theorem 6 . From the lemma, we have for any ordered partition $\left\{\tau_{k}\right\}$ of $[0,1]$ which refines $\left\{t_{j}, 1<j<N\right\}$ and any $\epsilon>0$

$$
\begin{aligned}
p\left\{x_{n} \in A\left(\left\{\tau_{k}\right\},-\epsilon / 3\right)\right\}- & p\left(x_{n},\left\{\tau_{k}\right\}, \epsilon / 3\right) \leqq p\left\{x_{n} \in A([0,1], 0)\right\} \\
& \leqq p\left\{x_{n} \in A\left(\left\{\tau_{k}\right\},+\epsilon / 3\right)\right\}+p\left(x_{n},\left\{\tau_{k}\right\}, \epsilon / 3\right) .
\end{aligned}
$$

From the corollary of Theorem 3 , for each $\epsilon>0$ and each partition $\left\{\tau_{k}\right\}$ there is an integer $n_{1}$ depending only on $\epsilon$ and the number of points in $\left\{\tau_{k}\right\}$ (i.e., independent of the values of the terms in each of the sequences $\left\{\alpha_{j}\right\}$, $\left\{\beta_{j}\right\},\left\{t_{j}\right\}$, and $\left.\left\{\tau_{j}\right\}\right)$ such that if $n \geqq n_{1}$

$$
\begin{aligned}
p\left\{x \in A\left(\left\{\tau_{k}\right\}, \pm \frac{\epsilon}{3}-\frac{\epsilon}{3}\right)\right\}-\frac{\epsilon}{3} \leqq & p\left\{x_{n} \in A\left(\left\{\tau_{k}\right\}, \pm \frac{\epsilon}{3}\right)\right\} \\
\leqq & \left\{x \in A\left(\left\{\tau_{k}\right\}, \pm \frac{\epsilon}{3}+\frac{\epsilon}{3}\right)\right\} \\
& +\frac{\epsilon}{3} .
\end{aligned}
$$

Finally, from the lemma again, for any partition $\left\{\tau_{k}\right\}$ the limiting process satisfies

$$
p\left\{x \in A\left(\left\{\tau_{k}\right\}, \frac{2 \epsilon}{3}\right)\right\} \leqq p\{x \in A([0,1], \epsilon)\}+p\left(x,\left\{\tau_{k}\right\}, \frac{\epsilon}{3}\right),
$$

and

$$
p\{x \in A([0,1],-\epsilon)\}-p\left(x,\left\{\tau_{k}\right\}, \frac{\epsilon}{3}\right) \leqq p\left\{x \in A\left(\left\{\tau_{k}\right\},-\frac{2 \epsilon}{3}\right)\right\} .
$$

To assemble (7.2), (7.3), (7.4) into a proof of $(7.1)^{\prime}$ choose $\left\{\tau_{k}\right\}$ so fine that (the limiting process having no fixed points of discontinuity)

$$
p\left(x,\left\{\tau_{k}\right\}, \epsilon / 3\right)<\epsilon / 3
$$

and for sufficiently large $n$, say $n \geqq n_{2}$,

$$
p\left(x_{n},\left\{\tau_{k}\right\}, \epsilon / 3\right)<\epsilon / 3 .
$$

The first choice is possible in view of the continuity properties of the limiting stochastic process, and the second follows from Theorem 6 . Let $n_{0}$ $=\max \left\{n_{1}, n_{2}\right\} ;$ then $n \geqq n_{0} \Rightarrow(7.3)$ holds for the partition $\left\{\tau_{k}\right\}$. Since these choices are made independent of the values of the terms in the sequences $\left\{\alpha_{j}\right\},\left\{\beta_{j}\right\},\left\{t_{j}\right\}$, and $\left\{\tau_{j}\right\}$, we have for each integer $N$ and each $\epsilon>0$ that 


$$
\begin{aligned}
p\{x \in A([0,1],-\epsilon)\}-\epsilon & \leqq p\left\{x \in A\left(\left\{\tau_{k}\right\},-\frac{2 \epsilon}{3}\right)\right\}-\frac{2 \epsilon}{3} \\
& \leqq p\left\{x_{n} \in A\left(\left\{\tau_{k}\right\},-\frac{\epsilon}{3}\right)\right\}-\frac{\epsilon}{3} \\
& \leqq p\left\{x_{n} \in A([0,1], 0)\right\}
\end{aligned}
$$

and similarly for

$$
p\{x \in A([0,1], \epsilon)\}+\epsilon \geqq p\left\{x_{n} \in A([0,1], 0)\right\}
$$

for any partition $t_{0}=0<t_{1}<\cdots<t_{N}=1$, and any $2 N$ numbers $\left\{\alpha_{1}, \cdots, \alpha_{N}, \beta_{1}, \cdots, \beta_{N}\right\}$. The necessity part of Theorem 7 is accordingly proved.

The sufficiency follows by choosing $N=3, \alpha_{0}=\alpha_{1}=\alpha_{2}=\alpha_{3}=-\infty, \beta_{0}=\beta_{3}$ $=+\infty, \beta_{1}=\beta_{2}=\lambda$ in (7.1), and applying monotone set convergence as $t_{1} \rightarrow t$ from below monotonically and $t_{2} \rightarrow t$ from above monotonically; (7.1) holds independent of the positions of $t_{1}$ and $t_{2}$, and hence for $n$ large

$$
p\left\{x_{n}(t) \leqq \lambda-\epsilon\right\}-\epsilon \leqq p\{x(t) \leqq \lambda\} \leqq p\left\{x_{n}(t) \leqq \lambda+\epsilon\right\}+\epsilon
$$

independent of $t$ (and $\lambda$ ). From Theorems 4 and 5 this establishes the uniform convergence in distribution of $\left\{x_{n}(t, \omega), t \in[0,1]\right\}$ to $\{x(t, \omega), t \in[0,1]\}$. This completes the proof of Theorem 7 .

In Theorem 9 of [3] a conclusion like that of Theorem 7 above was used to deduce the convergence in distribution of $\left\{F\left[x_{n}\right], n \geqq 1\right\}$ for a certain class of functionals $F$. In view of this, it certainly seems reasonable that our Theorem 7 could be used to obtain a restricted equivalence (at least) between the condition of uniform convergence in distribution of $\left\{x_{n}(t, \omega), t \in[0,1]\right\}$ and the convergence in distribution of $F\left[x_{n}\right]$ for all members of an appropriate class of functionals $F$. This question remains open; it appears to involve (considering the statement of Theorem 9 of [3]) some nontrivial topological subtleties.

3. Generalization of the Gnedenko-Kolmogorov theorems. In the present section the hypothesis of uniform convergence in distribution is used to obtain an extension of the results of [2] for the limiting distributions of sums of independent random variables. Partial results of this type were obtained in Theorem 5 of [3].

Let $\left\{x_{n k}, 1 \leqq k \leqq k_{n}, n \geqq 1\right\}$ be a sequence of finite sequences of independent random variables. Let $F_{n k}$ and $\phi_{n k}$ denote, respectively, the distribution and characteristic functions of $x_{n k}$. Let $\tau>0$ be chosen arbitrarily (and, once chosen, fixed for the rest of the discussion) and let

$$
\alpha_{n k}=\int_{|x|<t} x d F_{n k}(x) .
$$


For each $t \in[0,1]$, let

$$
\begin{aligned}
x_{n}(t, \omega) & =\sum\left\{x_{n k}: 1<k<t k_{n}\right\}, \\
\gamma_{n}(t) & =\sum\left\{\alpha_{n k}+\int_{-\infty}^{\infty} \frac{x}{1+x^{2}} d F_{n k}\left(x+\alpha_{n k}\right): 1<k<t k_{n}\right\}, \\
G_{n}(t ; x) & =\sum\left\{\int_{-\infty}^{\infty} \frac{u^{2}}{1+u^{2}} d F_{n k}\left(x+\alpha_{n k}\right): 1<k<t k_{n}\right\}
\end{aligned}
$$

where, in each case, $\sum\{\}$ denotes the sum of all members of the class specified in the braces. Let $\phi_{n}(t ; \mu)$ denote the characteristic function of $x_{n}(t, \omega)$. Assume that for any $\epsilon>0$

$$
\lim _{n \rightarrow \infty} \max _{1 \leqq k \leqq k_{n}} p\left\{\left|x_{n k}\right| \geqq \epsilon\right\}=0 .
$$

This last is the requirement that $\left\{x_{n k}\right\}$ be "infinitesimal" or, more descriptively, "asymptotically infinitesimal." The random variables $\left\{x_{n k}\right\}$ so constrained we shall call a "Poisson array." Finally, let, for any $h>0$

$$
G_{n}^{(h)}(t ; x)=\frac{1}{2 h} \int_{x-h}^{x+h} G_{n}(t ; u) d u .
$$

We remark, before proceeding with Theorem 8 , that if $\{x(t, \omega), t \in[0,1]\}$ has independent increments and no fixed points of discontinuity, and if $x(0, \omega) \equiv 0$, then the distribution of $x(t, \omega)$ is for each $t$ infinitely divisible and can be represented by a real number $\gamma(t)$ and a bounded nondecreasing function of $x G(t ; x)$. This pair $(\gamma(t), G(t ; x))$ will be called the Lévy-Khintchine representation (pair) of $x(t, \omega)$ (or of the distribution or characteristic function of $x(t, \omega))$. The representation is explicitly given by the Lévy-Khintchine formula

$$
\log E\{\exp i \mu x(t, \omega)\}=i \mu \gamma(t)+\int_{-\infty}^{\infty}\left(e^{i \mu x}-1-\frac{i \mu x}{1+x^{2}}\right) \frac{1+x^{2}}{x^{2}} d G(l ; x)
$$

wherein the integrand is assigned the value $-\mu^{2} / 2$ at $x=0$, by continuity.

TheOREM 8. The following conditions on a process sequence

$$
\left\{x_{n}(t, \omega), t \in[0,1]\right\}
$$

constructed as above from a Poisson array are equivalent:

(A) (i) $\exists \lim _{n \rightarrow \infty} \gamma_{n}(t)$ uniformly in $t \in[0,1]$.

(ii) $\exists \lim _{n \rightarrow \infty} G_{n}^{(h)}(t ; x)$ for each $h>0$, uniformly in $(t, x) \in[0,1]$ $\otimes[-\infty, \infty]$.

(B) There exist real functions $\gamma(t)$ and $G(t ; x)$ such that

(i') $\gamma(t)$ is continuous on $[0,1]$ and $\lim _{n \rightarrow \infty} \gamma_{n}(t)=\gamma(t)$ uniformly for $t \in[0,1]$. 
(ii') $G(t ; x)$ is jointly bounded and nondecreasing in $(t, x) \in[0,1]$ $\otimes(-\infty, \infty), G(t ; \infty)$ is continuous in $t \in[0,1], T(t,-\infty) \equiv 0$, and

$$
\lim _{n \rightarrow \infty} G_{n}(t ; x)=G(t ; x)
$$

for each $x$ which is a continuity point of $G(1 ; x)$, including $x= \pm \infty$, uniformly for $t \in[0,1]$ at each such $x$.

(C) $\left\{x_{n}(t, \omega), t \in[0,1]\right\}$ converges uniformly in distribution.

Moreover, under (any of) these conditions, the limiting process of (C) is a (separable) stochastic process with independent increments and no fixed points of discontinuity; the limits in (i) and (ii) of (A) are given by

$$
\left(\mathrm{i}^{\prime \prime}\right) \quad \lim _{n \rightarrow \infty} \gamma_{n}(t)=\gamma(t)
$$

$$
\text { (ii') } \lim _{n \rightarrow \infty} G_{n}^{(h)}(t ; x)=\frac{1}{2 h} \int_{x-h}^{x+h} G(t ; u) d u=G^{(h)}(t ; x)
$$

in terms of the function-pair $(\gamma, G)$ of $(\mathrm{B})$; and this function-pair is the LévyKhintchine representation pair for the limiting process of $(\mathrm{C})$.

Proof. 1. (A) $\Rightarrow(B)$. (i) and (i') are clearly equivalent, so it is only necessary to show that $(\mathrm{ii}) \Rightarrow\left(\mathrm{ii}^{\prime}\right)$. Let $\hat{G}^{(h)}(t ; x)$ be the limit in (ii). Clearly $\hat{G}^{(h)}(t ;-\infty)=0$. Let

$$
\begin{aligned}
& G^{* *}(t ; x)=\limsup _{n \rightarrow \infty} G_{n}(t ; x), \\
& G^{*}(t ; x)=\liminf _{n \rightarrow \infty} G_{n}(t ; x) .
\end{aligned}
$$

Since for any $h>0$ and any $(t, x)$,

$$
G_{n}^{(h)}(t ; x) \leqq G_{n}(t ; x+h)
$$

we have always

$$
G^{* *}(t ; x-h) \leqq \hat{G}^{(h)}(t ; x) \leqq G^{*}(t ; x+h) .
$$

Let $\epsilon>0$ be given. For each $h>0$ we can find $n_{\epsilon}$ so large that $n \geqq n_{\epsilon} \Rightarrow$ for all $(t, x) \in[0,1] \otimes[-\infty, \infty]$,

$$
\left|G_{n}^{(h)}(t ; x)-\hat{G}^{(h)}(t ; x)\right|<\epsilon .
$$

From (8.2) and (8.3) we conclude that for each $h>0, n \geqq n_{\epsilon}$, and any $(t, x)$ $\in[0,1] \otimes[-\infty, \infty]$,

$$
\begin{aligned}
G^{* *}(t ; x-2 h)-\epsilon & \leqq \hat{G}^{(h)}(t ; x-h)-\epsilon \leqq G_{n}^{(h)}(t ; x-h) \leqq G_{n}(t ; x) \\
& \leqq G_{n}^{(h)}(t ; x+h) \leqq \hat{G}^{(h)}(t ; x+h)+\epsilon \\
& \leqq G^{*}(t ; x+2 h)+\epsilon .
\end{aligned}
$$


Hence for any $h>0, \epsilon>0,(t, x) \in[0,1] \otimes[-\infty, \infty]$,

$$
G^{* *}(t ; x-2 h)-\epsilon \leqq G^{*}(t ; x) \leqq G^{* *}(t ; x) \leqq G^{*}(t ; x+2 h)+\epsilon .
$$

If we allow $\epsilon \rightarrow 0$ and $h \rightarrow 0$ we conclude that at every continuity point in $x$ of either $G^{*}(t ; x)$ or $G^{* *}(t ; x)$ we have

$$
\exists \lim _{n \rightarrow \infty} G_{n}(t ; x)=G^{*}(t ; x)=G^{* *}(t ; x)=G(t ; x),
$$

the last member of $(8.5)$ being a definition. Since $G^{*}(t ; x)$ and $G^{* *}(t ; x)$ are both nondecreasing in $x$ for each $t$, so is $G(t ; x)$ on its range of definition by (8.5). $G(t ; x)$ can therefore be defined for all real $x(|x|<\infty)$ by continuity from the right in $x$, in which case (8.5) holds for all real continuity points $x$ of $G(t ; x)$, for each $t$.

From this last, it follows easily that $G(t ; x)$ is jointly bounded and nondecreasing on $[0,1] \otimes(-\infty, \infty)$. Hence for any $t \in[0,1]$ and any real $x_{1}<x_{2}$,

$$
0 \leqq G\left(t ; x_{2}\right)-G\left(t ; x_{1}\right) \leqq G\left(1 ; x_{2}\right)-G\left(1 ; x_{1}\right) .
$$

Further, (8.4) can be rewritten as follows: for every $\epsilon>0$ and $h>0, n \geqq n_{\epsilon / 2}$ $\Rightarrow$ for all $(t, x)$

$$
G(t ; x-2 h)-\frac{\epsilon}{2} \leqq G_{n}(t ; x) \leqq G(t ; x+2 h)+\frac{\epsilon}{2} .
$$

If $x$ is a continuity point of $G(1 ; x)$ and $h_{0}$ is chosen so small that

$$
G\left(1 ; x+2 h_{0}\right)-G\left(1 ; x-2 h_{0}\right)<\frac{\epsilon}{2}
$$

then for this $x, \epsilon$, and $h_{0}, n \geqq n_{\epsilon / 2} \Rightarrow$ for all $t$,

$$
\begin{aligned}
\left|G_{n}(t ; x)-G(t ; x)\right| \leqq\left|G_{n}(t ; x)-G\left(t, x \pm 2 h_{0}\right)\right| \\
\quad+\left|G\left(t ; x+2 h_{0}\right)-G\left(t ; x-2 h_{0}\right)\right|<\frac{\epsilon}{2}+\frac{\epsilon}{2}=\epsilon .
\end{aligned}
$$

Hence (8.5) holds uniformly in $t$ for each $x$ which is a continuity point of $G(1 ; x)$.

From (8.7), letting $x \rightarrow \pm \infty$, it follows also that (8.5) holds uniformly in $t$ for $x= \pm \infty$.

It now remains to show that $G(t ;-\infty)=0$ and $G(t ;+\infty)$ is continuous for $t \in[0,1]$. The first statement follows easily from (8.7) by allowing $x \rightarrow-\infty$. The second statement follows less easily from the infinitesimality of the Poisson array $\left\{x_{n k}\right\}$. It is known [2] that the condition of infinitesimality on $\left\{x_{n k}\right\}$ implies that 
(8.8) $\quad \limsup _{n \rightarrow \infty}\left\{\left|G_{n}(t ; \infty)-G_{n}(s ; \infty)\right|:|t-s| \leqq \frac{1}{k_{n}}, 0 \leqq t, s \leqq 1\right\}=0$.

Let $\epsilon>0$ be given, and let $n_{1}$ be so large that for $n \geqq n_{1}$, we have

$$
\left|G_{n}(t ; \infty)-G_{n}(s ; \infty)\right|<\epsilon / 3
$$

for all $(t, s) \in[0,1]^{2}$ for which $|t-s|<1 / k_{n}$. Choose $n_{2}$ so large that if $n \geqq n_{2}$ we have

$$
\left|G_{n}(t ; \infty)-G(t ; \infty)\right|<\epsilon / 3
$$

for all $t \in[0,1]$ (since, as observed above, $G_{n}(t ; \infty)$ converges uniformly to $G(t ; \infty))$. Then if $n_{3}=\max \left\{n_{1}, n_{2}\right\}$, we have for any $t, s$ such that $|t-s|<k_{n_{3}}^{-1}$,

$$
\begin{aligned}
|G(t ; \infty)-G(s ; \infty)| \leqq & \left|G(t ; \infty)-G_{n_{3}}(t ; \infty)\right| \\
& +\left|G_{n_{3}}(t ; \infty)-G_{n_{3}}(s ; \infty)\right|+\left|G_{n_{3}}(s ; \infty)-G(s ; \infty)\right| \\
< & \frac{\epsilon}{3}+\frac{\epsilon}{3}+\frac{\epsilon}{3}=\epsilon .
\end{aligned}
$$

This establishes the continuity of $G(t ; \infty)$ on $[0,1]$ and concludes the proof that $(A) \Rightarrow(B)$.

2. (B) $\Rightarrow(\mathrm{A})$. We show that $\left(\mathrm{ii}^{\prime}\right) \Rightarrow(\mathrm{ii})$. Since $G_{n}(t ; x)$ for each $n$ and $G(t ; x)$ are all jointly bounded and nondecreasing in $(t, x)$, given $\epsilon>0$ we can find $\Lambda_{\epsilon}>0$ and a positive integer $n_{1}$ so large that if $n \geqq n_{1}$,

$$
\int_{|x|>\Lambda_{\epsilon}} d G_{n}(t ; x)<\frac{\epsilon}{4}
$$

and

$$
\int_{|x|>\Lambda_{\epsilon}} d G(t ; x)<\frac{\epsilon}{4} .
$$

Let $G_{0}$ be an upper bound on $G_{n}(t ; x)$ and $G(t ; x)$, and let $h>0$ be given.

Without loss of generality we may take $\pm \Lambda_{\epsilon}$ to be continuity points of $G(1 ; x)$. From Egoroff's theorem there exists a subset $C_{\epsilon}$ of $\left[-\Lambda_{\epsilon}, \Lambda_{\epsilon}\right]$, of Lebesgue measure less than $\epsilon h /\left(4 G_{0}\right)$ for which $G_{n}(t ; x) \rightarrow G(t ; x)$ uniformly on $[0,1] \otimes\left(\left[-\Lambda_{\epsilon}, \Lambda_{\epsilon}\right]-C_{\epsilon}\right)$; the set $C_{\epsilon}$ covers the discontinuity points of $G(1 ; x)$ in $\left[-\Lambda_{\epsilon}, \Lambda_{\epsilon}\right]$. Choose $n_{2}$ so large that $n \geqq n_{2} \Rightarrow$

$$
\left|G_{n}(t ; x)-G(t ; x)\right|<3 \epsilon / 4
$$

for all $(t, x) \in[0,1] \otimes\left[\left(-\Lambda_{\epsilon}, \Lambda_{\epsilon}\right]-C_{\epsilon}\right)$. Let $n_{3}$ be so large that $n \geqq n_{3} \Rightarrow$

$$
\left|G_{n}(t ; \infty)-G(t ; \infty)\right|<\epsilon / 4
$$

for all $t \in[0,1]$. Then if $n_{4}=\max \left\{n_{1}, n_{2}, n_{3}\right\}, n \geqq n_{4} \Rightarrow$ for all $t \in[0,1]$, 


$$
\begin{aligned}
\left|G_{n}(t ; x)-G(t ; x)\right| & <\frac{3 \epsilon}{4} \quad \text { if } x \in\left[-\Lambda_{\epsilon}, \Lambda_{\epsilon}\right]-C_{\epsilon}, \\
& \leqq \\
& \int_{|x|>\Lambda_{\epsilon}} d G_{n}(t ; x)+\int_{|x|>\Lambda_{\epsilon}} d G(t ; x) \\
& +\left|G_{n}(t ; \infty)-G(t ; \infty)\right| \\
& <\frac{\epsilon}{4}+\frac{\epsilon}{4}+\frac{\epsilon}{4}=\frac{3 \epsilon}{4} \quad \text { if }|x|>\Lambda_{\epsilon} .
\end{aligned}
$$

Hence if $n \geqq n_{4}$, for any $t$ we have

$$
\left|G_{n}(t ; x)-G(t ; x)\right|<\frac{3 \epsilon}{4} \quad \text { if } x \notin C_{\epsilon} .
$$

Let

$$
\hat{G}^{(h)}(t ; x)=\frac{1}{2 h} \int_{x-h}^{x+h} G(t ; u) d u ;
$$

we have then, for $n \geqq n_{4}$

$$
\begin{aligned}
\left|G_{n}^{(h)}(t ; x)-G^{(h)}(t ; x)\right| \leqq & \frac{1}{2 h} \int_{x-h}^{x+h}\left|G_{n}(t ; u)-G(t ; u)\right| d u \\
= & \frac{1}{2 h} \int_{|u-x| \leq h ; u \in C_{e}}\left|G_{n}(t ; u)-G(t ; u)\right| d u \\
& +\frac{1}{2 h} \int_{|u-x| \leq h ; u \notin C_{\epsilon}}\left|G_{n}(t ; u)-G(t ; u)\right| d u \\
& \leqq \frac{G_{0}}{h} \int_{u \in C_{\epsilon}} d u+\frac{3 \epsilon}{4} \\
& <\epsilon
\end{aligned}
$$

for any $(t, x) \in[0,1] \otimes[-\infty, \infty]$. Accordingly (ii) holds, $\hat{G}^{(h)}(t ; x)$ being the limit. $(B) \Rightarrow(A)$ is established.

3. (A) or $(B) \Rightarrow\left(\mathrm{i}^{\prime \prime}\right)$ and $\left(\mathrm{ii}^{\prime}\right)$ hold. ( $\left.\mathrm{i}^{\prime \prime}\right)$ follows trivially. (ii') follows with equal ease, since $(A)$ or $(B) \Rightarrow(B) \Rightarrow$

$$
\lim _{n \rightarrow \infty} G_{n}^{(h)}(t ; x)=\frac{1}{2 h} \int_{x-h}^{x+h} G(t ; u) d u .
$$

This is precisely (ii").

Before proceeding with the rest of the proof of Theorem 8 it is necessary to introduce some further notation: for the Poisson array $\left\{x_{n k}\right\}$, let 


$$
\begin{aligned}
\eta_{n}(t ; \mu) & =\sum\left\{i \mu \alpha_{n k}+\int_{-\infty}^{\infty}\left(e^{i \mu x}-1\right) d F_{n k}\left(x+\alpha_{n k}\right): 1<k<\left[t k_{n}\right]\right\} \\
& =i \mu \gamma_{n}(t)+\int_{-\infty}^{\infty}\left(e^{i \mu x}-1-\frac{i \mu x}{1+x^{2}}\right) \frac{1+x^{2}}{x^{2}} d_{x} G_{n}(t ; x)
\end{aligned}
$$

where as usual the integrand in the last member of the equality is assigned the value $-\mu^{2} / 2$ at $x=0$. Let also

$$
\Delta_{n}(t ; \mu)=\left|\log \phi_{n}(t ; \mu)-\eta_{n}(t ; \mu)\right| .
$$

The rest of the proof of Theorem 8 follows the methods of [2] for the analogous results for one-dimensional distributions, and we will regard these as well enough known that we may dispense with those details that carry over to the present case directly.

4. (B) or $(\mathrm{C}) \Rightarrow$ for any $M>0, \Delta_{n}(t ; \mu) \rightarrow 0$ uniformly for $(t ; \mu) \in[0,1]$ $\otimes[-M, M]:$ Let

$$
\begin{aligned}
& \phi_{n k}(\mu)=\int_{-\infty}^{\infty} e^{i \mu x} d F_{n k}(x) \\
& \hat{\phi}_{n k}(\mu)=\int_{-\infty}^{\infty} e^{i \mu x} d F_{n k}\left(x+\alpha_{n k}\right)
\end{aligned}
$$

Then

$$
\hat{\phi}_{n k}(\mu)=e^{-i \mu \alpha_{n k}} \phi_{n k}(\mu)
$$

and

$$
\phi_{n}(t ; \mu)=\prod\left\{\phi_{n k}(\mu): 1 \leqq k \leqq\left[t k_{n}\right]\right\}
$$

Expanding $\log \phi_{n}(t ; \mu)$ in powers of $\hat{\phi}_{n k}(\mu)-1$, we find that for large $n$ we have for any $(t, \mu) \in[0,1] \otimes[-M, M]$,

$$
\Delta_{n}(t ; \mu) \leqq \max _{1 \leqq k \leqq k_{n}} \frac{\left|\hat{\phi}_{n k}(\mu)-1\right|}{1-\left|\hat{\phi}_{n k}(\mu)-1\right|} \sum_{k=1}^{k_{n}}\left|\hat{\phi}_{n k}(\mu)-1\right| .
$$

There exist positive constants $C_{1}$ and $C_{2}$ depending only on $\tau$ (in the definition of the truncated means $\alpha_{n k}$ ) and $M$, for which for large $n$

$$
\begin{aligned}
\left|\hat{\phi}_{n k}(\mu)-1\right| & \leqq C_{1} \int_{-\infty}^{\infty} \frac{x^{2}}{1+x^{2}} d F_{n k}\left(x+\alpha_{n k}\right) \\
& \leqq C_{2} \cdot \frac{1}{M} \int_{0}^{M}|\log | \phi_{n k}(\mu)|| d \mu
\end{aligned}
$$

for all $|\mu| \leqq M$ and all $k=1,2, \cdots, k_{n}$. (8.9) and (8.10) hold if either (B) or (C) holds and $\left\{x_{n k}\right\}$ is an infinitesimal array. 
If either

$$
\sum_{k=1}^{k_{n}} \int_{-\infty}^{\infty} \frac{x^{2}}{1+x^{2}} d F_{n k}\left(x+\alpha_{n k}\right)=G_{n}(1 ; \infty)
$$

or

$$
\left|\prod_{k=1}^{k_{n}} \phi_{n k}(\mu)\right|^{-1}=\frac{1}{\left|\phi_{n}(1 ; \mu)\right|}
$$

is bounded uniformly for $|\mu| \leqq M$ for large $n$, the infinitesimality of the Poisson array $\left\{x_{n k}\right\}$ enforces $\Delta_{n}(t ; \mu) \rightarrow 0$ with the required uniformities.

If now (B) holds, (8.11) is bounded uniformly in $n$, from (ii'). If $(\mathrm{C})$ holds, $\boldsymbol{\phi} .(1 ; \mu)$ converges uniformly for $|\mu| \leqq M$ for any $M>0$ to $\phi(1 ; \mu)$ which must (again from the infinitesimality of $\left\{x_{n k}\right\}$; see [2]) be the characteristic function of an infinitely divisible distribution. Hence on any finite interval, $\phi(1 ; \mu)$ is bounded away from zero, and (8.12) is therefore bounded from above uniformly in $|\mu| \leqq M$ for large $n$. In either case, $\Delta_{n} \rightarrow 0$ as required.

5. $(\mathrm{A}) \Rightarrow(\mathrm{C})$, and the (separable) limiting process has independent increments and no fixed points of discontinuity; moreover, the limits $(\gamma, G)$ of (B) constitute the Lévy-Khintchine representation pair for the limiting process.

6. $(C) \Rightarrow(B)$, and the (separable) limiting process has independent increments and no fixed points of discontinuity; the Lévy-Khintchine representation for this limiting process is the pair $(\gamma, G)$ of $(\mathrm{B})$ : Let

$$
\phi(t ; \mu)=\lim _{n \rightarrow \infty} \phi_{n}(t ; \mu) ;
$$

the hypothesis here (condition (C) of Theorem 8) is precisely that the limit exists uniformly for $(t, \mu) \in[0,1] \otimes[-M, M]$ for any $M>0$. From the theory of limiting distributions for sums of independent random variables (see [2]) it follows that $\phi(t ; \mu)$ is for each $t$ the characteristic function of an infinitely divisible distribution; this (limiting) distribution is the distribution of the random variable $x(t ; \omega)$ from the limiting process. It has been shown that this limiting process has independent increments (Theorem 5 ).

Let $(G(t ; x), \gamma(t))$ be the Lévy-Khintchine representation pair for the distribution of $x(t, \omega)$. For each $t$, we then have

$$
\begin{aligned}
& \lim _{n \rightarrow \infty} G_{n}(t ; x)=G(t ; x), \\
& \lim _{n \rightarrow \infty} \gamma_{n}(t)=\gamma(t),
\end{aligned}
$$

the former relation holding for each $x$ which is a continuity point of $G(1 ; x)$ and $x= \pm \infty$. We require that these limits satisfy the conditions of (B).

First we recall that the limiting process has independent increments, so 
that $G(t ; x)$ is bounded and jointly nondecreasing in $(t, x)$. Further the condition of infinitesimality on the Poisson array is precisely $(6.1)$ " of the corollary to Theorem 6 . Here $\hat{\delta}_{n}$ may be taken to be any sequence which is $0\left(k_{n}^{-1}\right)$. Hence the limiting process is without fixed points of discontinuity, and $G(t ; x)$ must therefore satisfy the continuity conditions of (B). Clearly $\gamma(t)$ is continuous, too.

Let $x$ be a fixed continuity point of $G(1 ; x)$, possibly $\pm \infty$. Then $x$ must also be a continuity point of $G(t ; x)$ for every $t \in[0,1]$ and $\lim _{n} G_{n}(t ; x)$ $=G(t ; x)$ for all $t \in[0,1]$, from $(8.16) . G(t ; x)$ is continuous and nondecreasing in $t$ and for each $n, G_{n}(t ; x)$ is nondecreasing in $t$. From a theorem of Dini it follows that $\lim _{n} G_{n}(t ; x)=G(t ; x)$ uniformly for $t \in[0,1]$. This completes the proof of Part 6 , since we have shown that $(C) \Rightarrow(B)$, and that $\{x(t, \omega), t \in[0,1]\}$, the limiting process, has independent increments and no fixed points of discontinuity with Lévy-Khintchine representation pair $(G, \gamma)$.

This completes the proof of Theorem 8 . The theorem can obviously be restated in terms of the alternative Lévy representations of the limiting process; we omit this and bring this paper to its conclusion.

\section{REFERENCES}

1. J. Doob, Stochastic processes, New York, Wiley, 1953.

2. B. V. Gnedenko and A. N. Kolmogorov, Limit distribution for sums of independent random variables, Cambridge, Mass., Addison-Wesley, 1954.

3. E. G. Kimme, On the convergence of sequences of stochastic processes, Trans. Amer. Math. Soc. vol. 84 (1957) pp. 208-229.

4. M. Loève, Probability theory, Toronto-New York-London, Van Nostrand, 1955.

Bell Telephone Laboratories, Inc., Murray Hill, New Jersey 